\section{A CASE OF PRIMARY BILIARY CIRRHOSIS}

BY

\section{H. G. H. RICHARDS, M.B., M.R.C.P.Ed Consultant Pathologist}

AND

\section{B. S. FULLER, M.D., M.R.C.P. Consultant Physician \\ Royal Hampshire County Hospital, Winchester}

Primary biliary cirrhosis is a rare condition, which is our reason for recording the following case.

In an analysis of the literature Ahrens et al. (1950) gave a series of 22 cases of biliary cirrhosis, of which 17 were proved to be of the primary type. They also reviewed all cases of chronic biliary obstruction with generalized xanthomatosis reported since 1851 . These cases numbered 92 , but in only 49 of these was the seat of the biliary obstruction confirmed by surgical operation, necropsy, or both. The seat of the obstruction was found to be extrahepatic in 24 cases and intrahepatic in 25. Dr. Thomas Parkinson (1952) showed a further case of primary biliary cirrhosis at the Royal Society of Medicine in March, 1952. In this case it was confirmed by laparotomy that the obstruction was intrahepatic. This patient had, however, xanthomatosis.

The aetiology of this disease is at present unknown, but adult women are predominantly affected. The biliary obstruction is intrahepatic and is not associated with previous liver disease. The onset is insidious and the course prolonged. It is characterized by general pruritus, jaundice, good nutritional condition, massive enlargement of the liver, splenomegaly, hyperpigmentation, and coarseness of the skin. A large proportion of the cases develop generalized xanthomatosis, probably the result of prolonged hypercholesterolaemia.

\section{Case History}

A married woman aged 40 with no children was referred to one of us by her doctor on December 19, 1951, with a history of generalized pruritus. Twelve months previously she had sustained a wedge fracture of the eleventh dorsal vertebra in a car accident. She was put in a plaster jacket and had troublesome pruritus under the plaster. When the plaster jacket was removed the pruritus persisted, and she noticed that it was generalized, even involving the soles of her feet. She had a little flatulent dyspepsia, but was not intolerant to fat. Her urine stained her clothes just recently. Catamenia was regular 2-3/28.

On examination she was seen to be of spare build; weight $108 \mathrm{lb}$. (49 kg.). A slight icteric tinge of the skin and conjunctivae was present. There were no xanthomata of the skin. The lungs and cardiovascular system were normal. The liver edge could be felt 1 in. $(2.5 \mathrm{~cm}$.) below the costal margin, but was not tender. The tip of the spleen could also be felt below the costal margin. The icterus was not noticed by her doctor when he saw her a few days previously. She was admitted for investigation. The results of the pathological investigations are shown in the Table.

A laparotomy, to settle whether the obstruction was extrahepatic or intrahepatic, showed that the liver was considerably enlarged, especially the left lobe, and the spleen slightly enlarged; the gall-bladder, the extrahepatic biliary tract, and the pancreas were all normal. A fragment of the liver was taken for biopsy.

Histology of Liver.- " The normal lobular pattern is well maintained and the vast majority of parenchymal cells are unremarkable. There is little evidence of parenchymal regeneration. The striking lesion lies in the portal tracts. They are conspicuous and enlarged by well-marked fibrosis and dense infiltrations by mobile cells-partly lymphocytes, but in places polymorphonuclears. The bile ducts have disappeared from some tracts or are so swamped by mobile cells as to be unrecognizable. Where identified they are encompassed, and seem constricted by their surroundings, although there is no sign whatever of inflammatory exudation into their lumen. One further interesting feature : dense cellular portal-tract infiltrations in places have cut

Results of Pathological Investigations

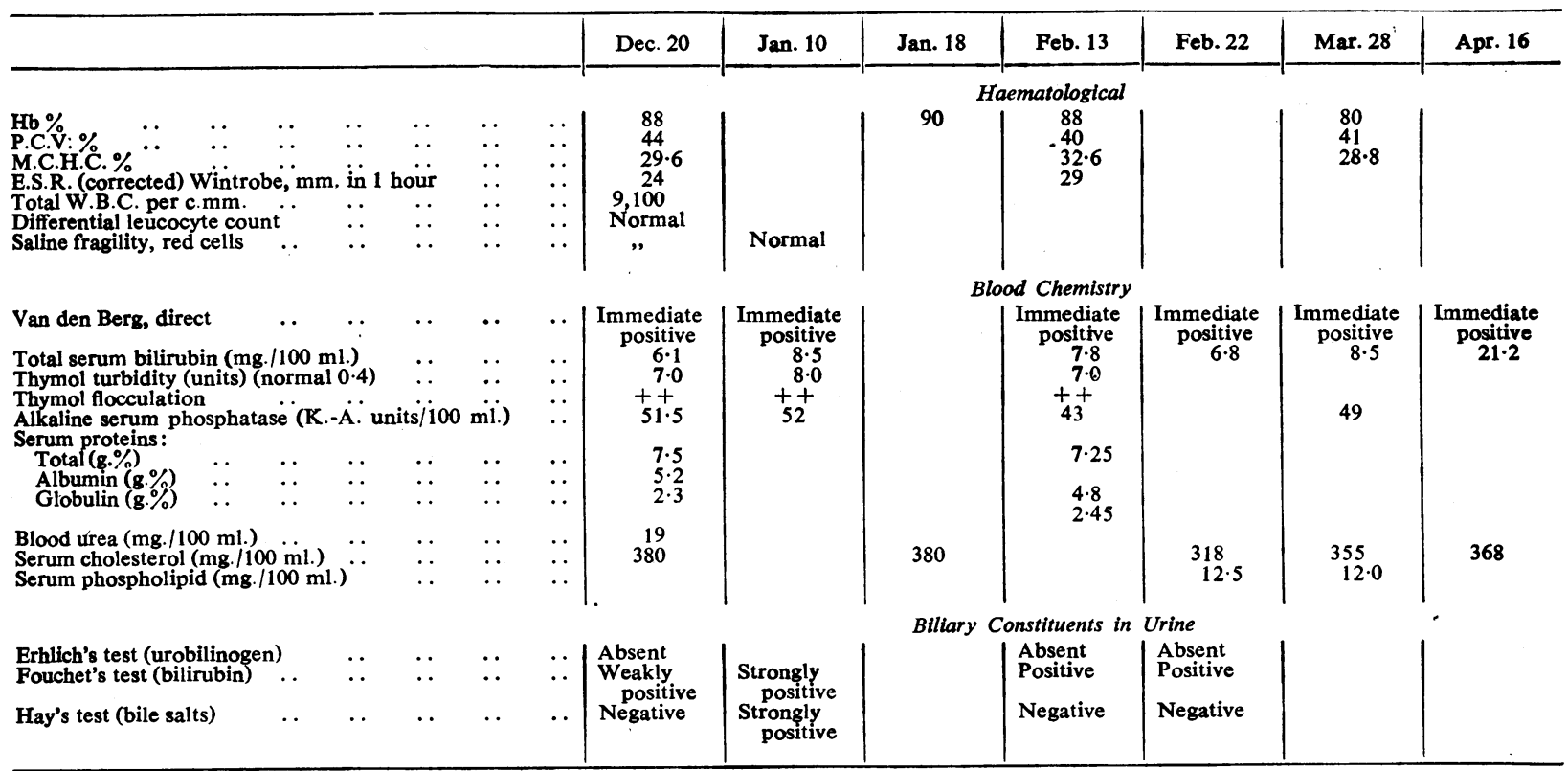


off groups of peripheral zone cells from adjacent lobules and are surrounding them. These features are characteristic of primary biliary cirrhosis and long-standing obstructive jaundice of all aetiologies, whether intrahepatic or extrahepatic."

The patient made an uninterrupted recovery and left hospital five weeks later with her jaundice much diminished and her pruritus hardly worrying her. She was given a three-weeks course of streptomycin, $1 \mathrm{~g}$. daily, vitamins, and a full diet.

The pruritus returned rather severely in April, 1952, disturbing her sleep, and the jaundice increased. She was seen by Dr. Sheila Sherlock at the Postgraduate Medical School of London, who advised testosterone propionate, $25 \mathrm{mg}$., sublingually daily, and an increase in the vitamin replacement therapy. The pruritus was quickly relieved, and the patient kept well until August, when on holiday. On admission to Hammersmith Hospital she was found to be severely anaemic, having had haemorrhage from her alimentary tract.

\section{Summary}

A rare disease is described in which the differential diagnosis can be settled only by an exploratory laparotomy and a biopsy of the liver.

Little is known of the aetiology, and at present treatment is unfortunately purely symptomatic. It is interesting to note that usually the condition arises in women round about the age of 40 , which suggests the possibility of an endocrine factor.

The prognosis is gloomy in that there has been no recorded case of recovery, and the disease proceeds on a relentless course with occasional intermissions. Death may occur after only two years, although Ahrens et al. record that patients of theirs are still alive after eleven years.

We are indebted to Mr. J. Troup, who undertook the exploratory laparotomy and the biopsy of the liver which enabled the diagnosis to be established.

\section{Refrerences}

Ahrens, E. H., jun., Payne, M. A., Kunkel, H. G., Eisenmenger, W. J., and Blondheim, S. H. (1950). Medicine, 29, 299

Parkinson, T. (1952). Proc. roy. Soc. Med., 45, 150.

\section{Medical Memorandum}

\section{A Case of Acute Diphenhydramine Hydrochloride Poisoning}

The purpose of this article is twofold-to draw attention once again to the danger which antihistamines hold for young children, should the container be left lying about, and to point out that the naked-eye appearances at necropsy can simulate those of bronchopneumonia. A mistaken report of bronchopneumonia could arise if no histological sections were to hand, and if for any reason it were not known that a harmful substance had been eaten.

\section{CAse Report}

A mother, coming downstairs at 4 p.m. one day after an absence of about one hour, found a spilled bottle and several pifls scattered about the floor. During her absence her youngest son, a perfectly healthy child of 2 years 7 months, had been caught eating some of the pills by an elder brother. The number of pills in the bottle before the incident was unknown, and so the number actually eaten was never discovered. The child ate a normal meal at about 5 p.m., and continued apparently perfectly well until shortly after being put to bed at 6.15 p.m. He then began to roll his eyes and wave his arms about. His condition rapidly worsened ; he became extremely excitable, and began having "fits." He was then removed to hospital, where he was noted on admission at 7.30 p.m. to be extremely cyanosed, and to be having repeated convulsions. The stomach was washed out once or twice and oxygen administered. Death occurred at 8.40 p.m. the same evening.

Post-mortem Findings.-At the time the necropsy was performed it had been incorrectly reported that the child had swallowed a few calcium gluconate tablets, which would, of course, have no harmful effects. There were scattered Tardieu's spots over the visceral pleura of both lungs. The trachea was somewhat congested, and contained a small quantity of blood-stained watery fluid. The lungs themselves presented a striking appearance: dark-purple depressed areas alternated with paler emphysematous areas, but portions of the dark areas sank in water. Apart from a few flecks of white powder adhering to the mucous membrane of the stomach the gastro-intestinal tract was normal. Both the cardiovascular and the urogenital systems were normal. The liver had moderate cloudy swelling only. The spleen showed some venous congestion. It was thought at this stage, on the basis of these naked-eye appearances, that death had resulted from bronchopneumonia. Microscopically, the brain showed capillaries somewhat dilated, and in the lungs the dark-purple areas were seen to be due to massive haemorrhages into the interalveolar spaces and the alveolar lumina. There was a moderate desquamation of the epithelium of the smaller bronchioles. The lighter areas showed a patchy emphysema. The myocardium, kidney, and liver showed only a slight cloudy swelling.

Chemical Analysis.-This showed the presence of a small quantity of a basic substance giving the chemical reactions of diphenhydramine hydrochloride (" benadryl ") in samples of blood, urine, and stomach washings ; it was also isolated from the intestines, stomach, brain, liver, lungs, spleen, heart, and kidney. The total amount present was small, and was contaminated with other material, so that no opinion could be given of the amount of diphenhydramine hydrochloride absorbed.

\section{COMMENT}

In both clinical and pathological aspects this case appears to have followed the pattern of the cases reported by Tobias (1949) and by Miller and Pedley (1950). The onset of symptoms was delayed by about two and a half hours, and survival lasted about four hours after the taking of the drug. Although there was little catarrhal discharge, the mode of death was clearly asphyxial, as demonstrated by convulsions and cyanosis, Tardieu's spots, and lung lesions found after death. Such a severe haemorrhagic manifestation in the lung, and, with the possible exception of the spleen, not marked elsewhere, suggests the lung as the susceptible organ.

Although no mechanism has yet been suggested to account for the poisonous effects of antihistaminic compounds, it is perhaps interesting that the salient pathological lesion, an interalveolar and intra-alveolar haemorrhagic oedema of the lung, is extremely suggestive of a histaminic effect. Histamine and antihistamines are able to induce or influence variations in capillary diameter and permeability, and it is not difficult to imagine lesions such as the one described arising from some imbalance between their tissue concentrations.

I wish to thank Mr. L. C. Nicholl, of the Forensic Science Laboratory, Wakefield, for permission to publish his report on the chemical analysis.

\section{F. E. Aaron, M.B., D.C.P., Formerly Registrar in Pathology,} Royal Halifax Infirmary.

Miller, A. A., and Pedley, E. (1950). Britlsh Medical Journal, 1, 1115. Tobias, M. (1949), Ibid., 1, 1098 